\title{
Montagegerechte Gestaltung verfahrenstechnischer Anlagen
}

\author{
Eberhard Wegener
}

Verfahrenstechnische Anlagen werden in erster Linie unter dem Aspekt der Funktionserfuillung entwickelt, bei ihrer konstruktiven Durchbildung (Gestaltung) müssen zusätzlich die Anforderungen aus den Rekonstruktionsphasen Montage - Betrieb - Instandhaltung - Rekonstruktion berïcksichtigt werden. Nachfolgend werden die Möglichkeiten einer montagegerechten Gestaltung behandelt, die in der Planungsphase zu schaffen ist. Es reicht jedoch nicht aus, die prinzipielle Montierbarkeit einer Anlage nachzuweisen, sondern es sind Untersuchungen zur effektiven Gestaltung des Montageprozesses durchzufuihren.

\section{Zur Begründung des Untersuchungs- bereiches}

Aus montagetechnologischer Sicht wird die Anlage durch ihre Montageeinheiten und deren geometrische Anordnung zueinander sowie durch die örtlichen Bedingungen der konkreten Arbeitsumwelt gekennzeichnet. Diese Faktoren bestimmen daher die technische Durchfuihrung der Montage wesentlich, da in der Gestaltungslösung Umfang, Schwierigkeitsgrad, einsetzbare Montagetechnologien usw. im Voraus bestimmt und eingegrenzt werden. Versäumnisse, Fehler und Mängel, die in den zeitlich vorgelagerten Bearbeitungsstufen entstehen, beeinflussen den Montageumfang ebenfalls. Sie wirken sich hemmend und kostenverursachend aus und müssen in der Montage beseitigt werden. Die Berüicksichtigung einer montagegerechten Gestaltungslösung in der Planungsphase wird durch folgende Faktoren erschwert:

- Die Arbeitsteilung in Planung und Realisierung bis hin zur Vergabe von reinen Montageleistungen erfordert vom Planer ausreichende Kenntnis über mögliche Montagetechnologien/-technik und über die die Montage beeinflussenden Faktoren, über die im allgemeinen erst der erfahrene Projektant verfügt.

- Es erfolgt kaum eine Vermittlung von Kenntnissen über die Anlagenmontage in der Ausbildung von Ingenieuren infolge der speziellen und komplizierten Thematik.

- Aus gleichem Grund existiert kaum Fachliteratur zu diesem Gebiet, wenn von Zauchau [1] und speziellen wissenschaftlichen Veröffentlichungen abgesehen wird, denn auch in den den Anlagenbau behandelnden Werken wie z. B. Bernecker [2], Ullrich [3], May [4], Reichert [5] oder in [6] werden nur Teilprobleme angeschnitten.

Die begrenzte Voraussicht über den erforderlichen Informationsbedarf des weniger erfahrenen Planers und das Vorhandensein sog. „Informationsbarrieren“ (Engel- bert [7]), die logischerweise auftreten, wenn allein die Einflussgrößen auf eine montagegerechte Ausrüstungsanordnung (Abbildung 1) betrachtet werden, führen daher zu - aus der Sicht der Montage - unvollständigen Gestaltungslösungen. Als Informationsbarrieren treten auf die

- Bewusstheitsbarriere (Beeinflussung des Informationsbedarfes durch Motivation, Arbeitsgewohnheiten und Arbeitsumgebung),

- Resonanzbarriere (Nichtnutzung vorhandener Informationen z. B. aufgrund mangelnder Berufserfahrung oder Voreingenommenheit),

- Kommunikationsbarriere (unvollständige Widerspiegelung vorliegender Sachverhalte).

Notwendige Schritte im Planungsablauf zur Erzielung einer montagegerechten Anlagengestaltung werden nachstehend kurz behandelt.

\section{Der Einfluss der Anlagenplanung auf den Montageprozess}

Die Anlagenplanung selbst ist ein Stufenprozess. Mit dem Voranschreiten der Planung nehmen in den einzelnen Stufen Umfang und Detailliertheit der verfuigbaren Informationen und damit die Einflussnahme - auch der Montage - auf die Gestaltungslösung zu.

Der Arbeitsgegenstand - hier die zu montierende Ausrüstung oder Teile hiervon (Montageeinheiten) - ist Träger der Montagegerechtheit. Die Montageeinheit wird charakterisiert durch:

- ihre Masse,

- ihre Abmessungen und geometrische Gestaltung, die Lage des Schwerpunktes und die Anschlagmöglichkeiten für Lastaufnahmemittel,

- ihren Anlieferungszustand (Komplettierungsgrad, Kompliziertheit, Empfindlichkeit),

- ihre Stabilität,

- die Anzahl, Art und Lage der Fügeflächen und der vorgesehenen Verbindungsarten.

Eine Erhöhung der Montageeffektivität infolge planerisch/konstruktiver Lösungen ist erreichbar durch:

- die Verringerung der zu montierenden Bauelementezahl insgesamt (absolut),

- die Verringerung der auf der Baustelle zu montierenden Bauelementezahl (relativ),

- eine Verringerung der Ausrüistungsvielfalt,

- die Anpassung der Montageeinheiten an die erforderlichen Montageverrichtungen/-operationen,

- die Beriicksichtigung des Sonderlastfalles „Montage“. 


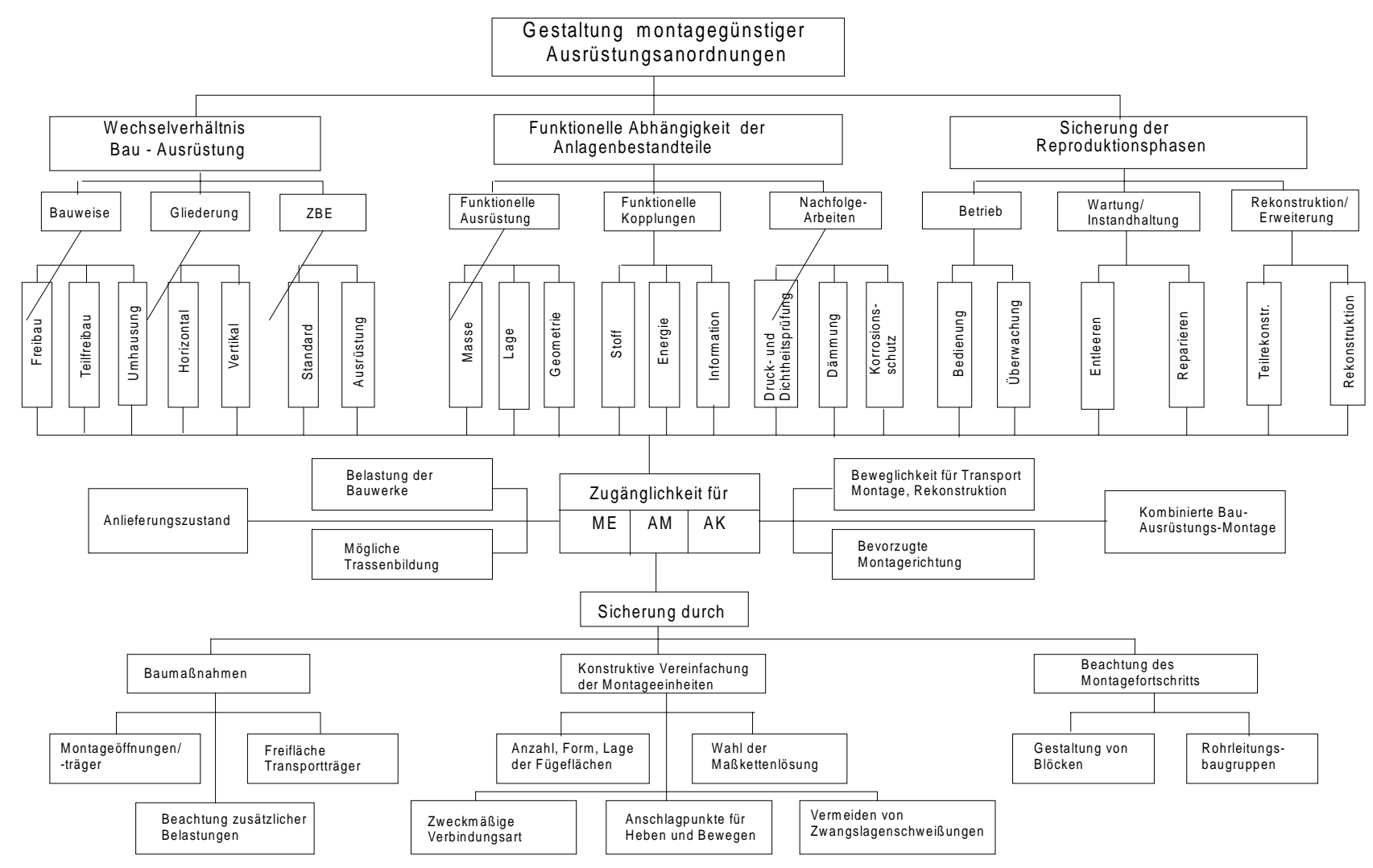

Abb. 1: Einflussgrößen auf die Gestaltung montagegerechter Ausrüstungsanordnungen

Im weiteren Sinne fuihrt eine Anpassung der Anpassung der Arbeitsmittel, der Arbeitsumwelt, der Arbeitskraft, der Montageorganisation sowie der Montagebedingungen zu einer Verbesserung der Montageeffektivität (Abbildung 2 [7]), in unterschiedlichem Maße in den einzelnen Planungsstufen.

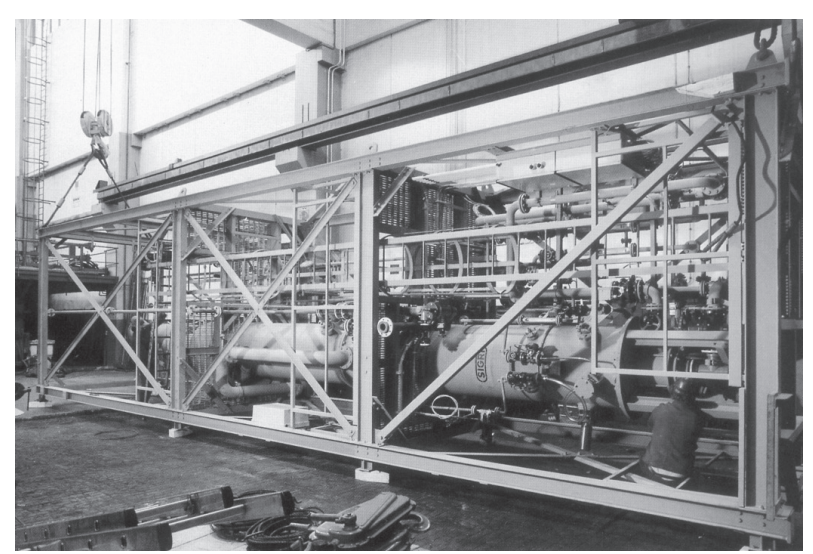

Abb. 2: Vormontierte Syntheseanlage ( package unit), Gebr. Sulzer AG Winterthur.

Allgemeine Aussagen sind:

- Eine Verringerung der Bauelementezahl insgesamt ist montagetechnologisch nicht beeinflussbar, Art und Anzahl der Ausrüstungen werden verfahrenstechnisch aus funktioneller Sicht festgelegt. Dem Process Engineering ist daher die Aufgabe gestellt, mehrere als notwendig erkannte Teilfunktionen in einen einzigen Funktionsträger zusammen zu fassen, d. h. eine Integralbauweise anzustreben (Kombination von Wirkprinzipien).

- Eine Verringerung der auf der Baustelle zu montierenden Anzahl von Bauelementen wird durch die
Verlegung von Montageverrichtungen/-operationen in den dem Endmontageort vorgelagerten Montageprozessstufen (Fertigungswerkstatt, Vormontageplatz) erreicht durch sog. Aggregation. Hier erfolgt eine Vergrößerung der Masse und Maße sowie eine Veränderung der geometrischen Form der Montageeinheit als konstruktives Prinzip. Die Anzahl der insgesamt zur Montageeinheit gehörenden Bauelementezahl ändert sich nicht, der Arbeitsumfang am Endmontageort jedoch beträchtlich. Diese Aggregation (Blockbildung, package units) mit weitestgehender Komplettierung stellt hohe Anforderungen an die konstruktive Durchbildung und die Organisation der Fertigung infolge der Vielfalt beteiligter Gewerke. Abbildungen 2 bis 5 zeigen eine vormontierte Syntheseanlage einschließlich ihrem Antransport und ihrer Montage.

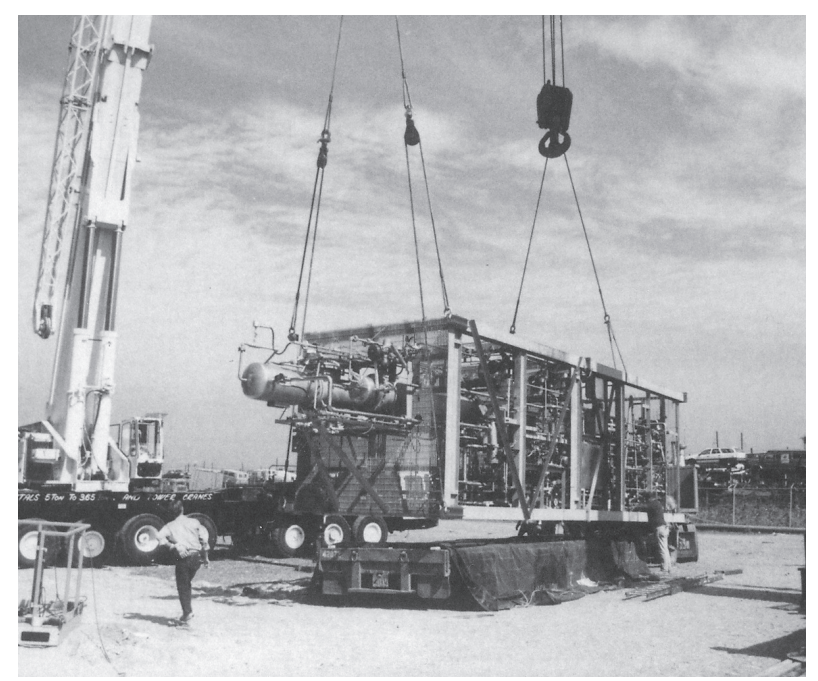

Abb. 3: Antransport des Blockes 


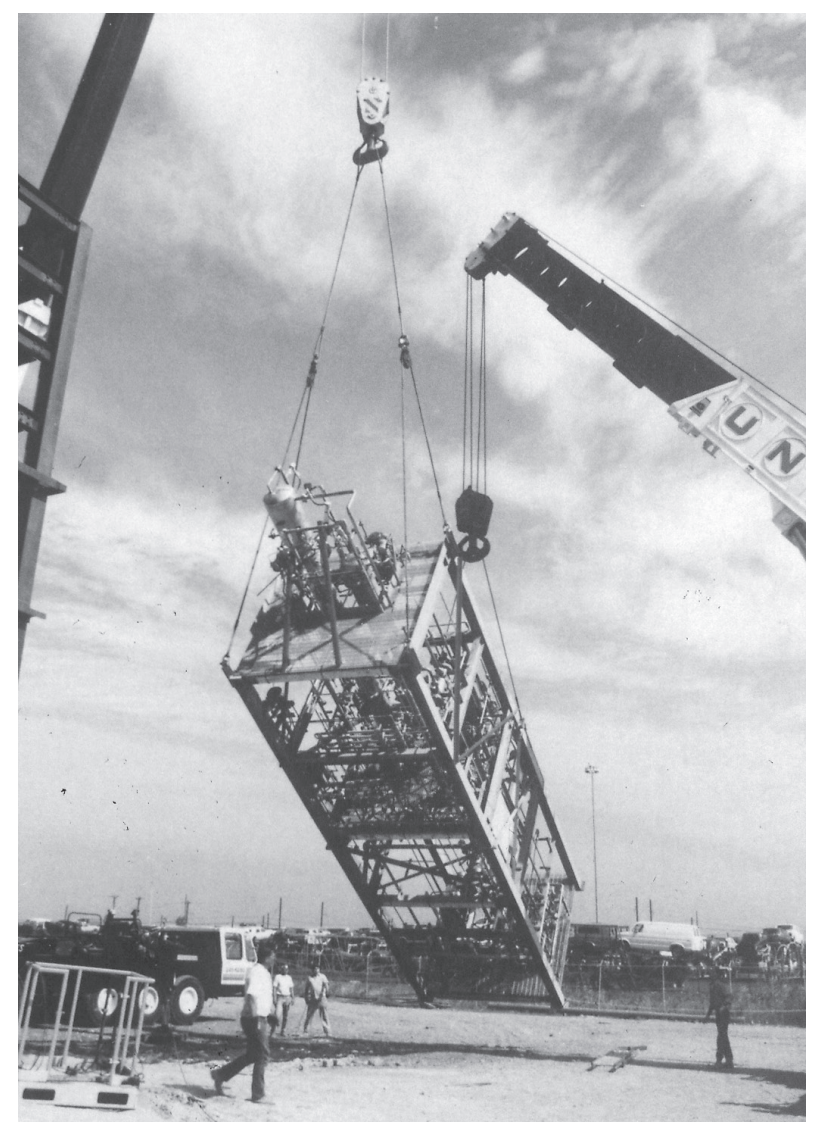

Abb. 4: Herstellen der Einbaulage

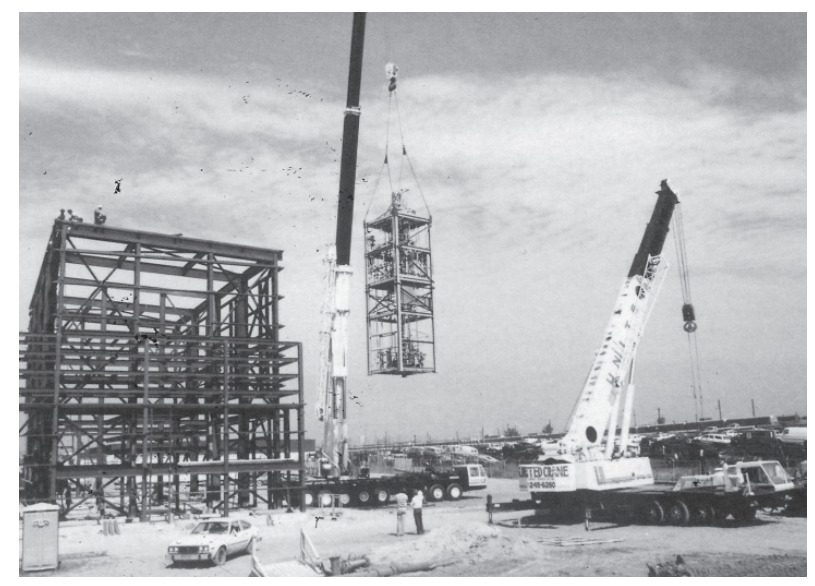

Abb. 5: Einbringen zum Endmontageort

Für die Rohrleitungsvorfertigung bedeutet das die sinnvolle Zerlegung von Rohrsträngen in fertigungsgerechte, transportfähige und montagegerechte Rohrleitungsbauteile und -baugruppen. Die Anpassung der Montageeinheit an Randbedingungen zur Aufwandsreduzierung fuihrt zu Kompromisslösungen in folgender Hinsicht:

- Anpassung an die Arbeitsmittel: Festlegung der Masse/Geometrie so, dass eine max. Auslastung der Hebezeugparameter erfolgt, möglichst Eigenstabilität vorliegt, eine definierte Schwerpunktlage gegeben ist und erforderliche Anschlagpunkte für die zweckmäßigsten Lastaufnahmemittel vorhanden sind.

- Anpassung an die konkrete Arbeitsumwelt: Festlegung der Masse/Abmessungen/Geometrie so, dass ein behinderungsfreies Zuführen, Positionieren, Lagesichern, Fügen und Nachbereiten unter Berücksichtigung der Zugänglichkeit und des Montagefortschrittes gewährleistet werden.
- Anpassung an die Montageverrichtungen und -operationen: Gestaltung der Anzahl, Art und Lage der Fügeflächen so, dass rationelle Fügeverfahren einsetzbar sind, notwendige Montageverrichtungen/-operationen auf ein Minimum beschränkt werden sowie Prüf- und Kontrollprozesse rationalisiert werden können. Dabei sind gleichzeitig die Beschränkungen, die sich aus dem Montagefortschritt und der damit veränderten Einbausituation ergeben, zu beachten.

- Die Montageorganisation selbst hat maßgebenden Einfluss auf die Effektivität der Montage. Zielstellung ist die Sicherung von Parallelität, Kontinuität, Gleichmäßigkeit und Proportionalität im Montageablauf. Während Kontinuität, Proportionalität und Gleichmäßigkeit durch organisatorische Maßnahmen gewährleistet werden, ist eine mögliche Parallelarbeit von Montagearbeiten von der gestalterischen Lösung abhängig. Infolge von Arbeitsteilung und Spezialisierung innerhalb des Montageprozesses sind die Voraussetzungen gegeben, dass mehrere Teilprozesse gleichzeitig und räumlich nebeneinander durchgefuihrt werden können. Die entscheidende Grenze ist die optimale Arbeitsdichte. Sie wird überschritten, wenn es zur gegenseitigen Behinderung der Arbeitskräfte oder Arbeitsmittel kommt. Die mögliche Arbeitsdichte hängt damit auch von der Gestaltung der Anlage und der konstruktiven Durchbildung der Montageeinheit ab (Abbildung 6).

Unter Beachtung der Kompliziertheit der Montageeinheit, Vermeidung möglicher Behinderungen, Gewährleistung der Lagesicherung und Vermeidung von Beschädigungen und Demontagen soll die Gestaltungslösung eine eindeutige, zeitabhängige Ablaufplanung ermöglichen. Diese Zeitabhängigkeit wird durch Vorlaufbedingungen, Bedingungen der Gleichzeitigkeit und Be-

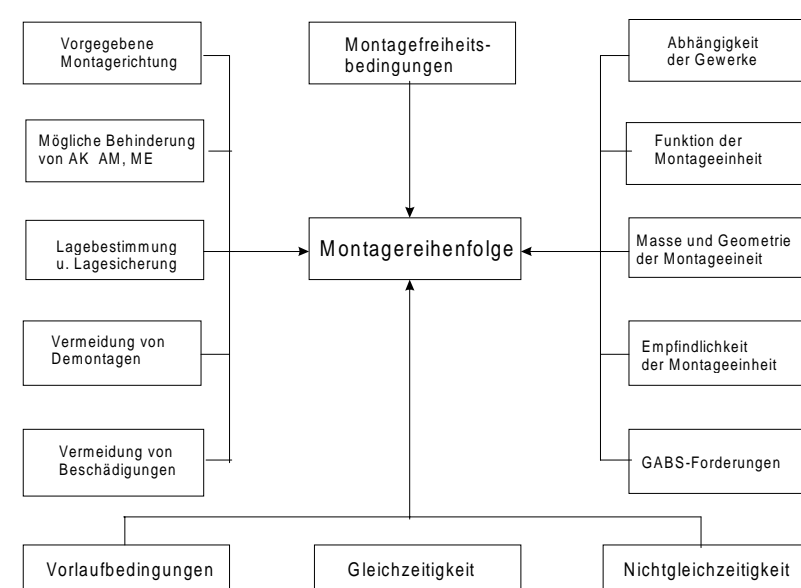

Abb. 7: Einflüsse auf die Montagereihenfolge

dingungen der Nichtgleichzeitigkeit ausgedrückt, d. h. die Montagereihenfolge ist nicht frei wählbar (Abb. 7).

- Der Lastfall Montage als Sonderlastfall ist insofern bedeutungsvoll, dass ein wesentlicher Anteil der tatsächlichen Kräfte und Momente an der Montageeinheit nicht der Funktionserfuillung dient, sondern sich aus den gleichzeitig auftretenden Belastungsgrößen und der Abfolge verschiedener Belastungszu- 


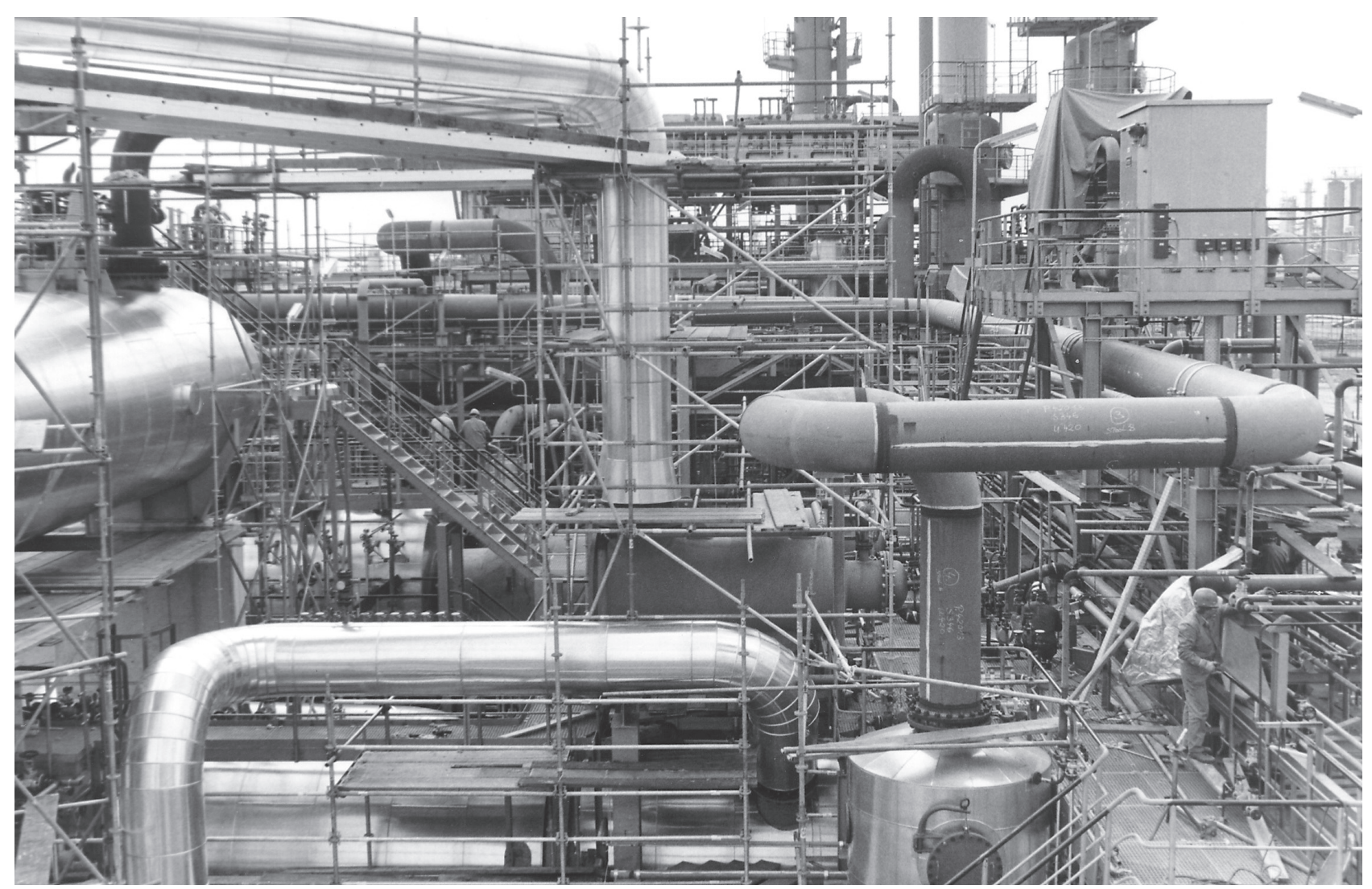

Bild 6: Montagefortschritt und Arbeitsdichte (PCK Schwedt AG)

stände für den Bewegungszustand vom Ausgangsort bis zum Einbauort und während des Herstellens der Einbaulage und während des Fügens bzw. Nachbereitens dient. Die Vielfalt der Formen und Belastungen zwingt häufig zu ingenieurmäßigen Näherungen in der Festigkeits- und Stabilitätsberechnung.

\section{Zuordnung der Rationalisierungs- möglichkeiten zu den Planungsstufen}

Die einzelnen Möglichkeiten zur Schaffung einer montagegerechten Anlagengestaltung mit der Zuordnung auf die Planungsstufen, vom process-engineering bis hin zum basic- bzw. detail-engineering, enthält Tab. 1, wobei eine Beurteilung der Gestaltungslösung hinsichtlich ihrer Monatagegerechtheit ebenfalls stufenweise erfolgen muss, um folgerichtig die Anforderungen an eine effektive Montagedurchfuihrung zu berücksichtigen und auf dem jeweiligen Konkretisierungsniveau der Planungsunterlagen zu gewährleisten, z. B. durch CheckListen. Die Probleme der Lösungsbewertung - die Montagegerechtheit ist ja nur eine Teilqualität der Gesamtlösung - sind Gegenstand eines weiteren Beitrages.

\section{Literaturverzeichnis}

[1] Zauchau, H.: Außenmontage im Maschinen- und Anlagenbau, Verlag Technik, Berlin 1982

[2] Bernecker, G.: Planung und Bau verfahrenstechnischer Anlagen, VDI Verlag, Düsseldorf 1990
[3] Ullrich, H.: Hochschulkurs IV Anlagenbau. Verfahrenstechnik 14 (1980) 11, 15 (1981) 1, 15 (1981) 3, 15 (1981) 5, 15 (1981) 7, 15 (1981) 9, Beilage

[4] May, H.: Anlagen-Projektierung in der Verfahrensindustrie. Dr. Alfred Huithig-Verlag, Heidelberg 1974

[5] Reichert, O.: Systematische Planung von Anlagen der Verfahrenstechnik. Carl Hanser Verlag, München Wien 1978

[6] Ullmans Encyklopädie der technischen Chemie, Bd. 4: Verfahrensentwicklung und Planung von Anlagen. Verlag Chemie, Weinheim 1974

[7] Engelbert, H.: Informationsbarrieren Informatik 21 (1974) 5, S. 51-54 und 21 (1974) 6, S. 47-51

[8] Wegener, E.: Verständigungsgerecht? Bemerkungen zum Begriff ,... gerecht“ am Beispiel der Montagegerechtheit im Apparate- und Anlagenbau. Wissenschaftliche Beiträge der Technischen Fachhochschule Wildau 2/2000

[9] Porteé, R.: Die Montage verfahrenstechnischer Anlagen, Einfluisse und Ablauf. Technische Fachhochschule Wildau, Fachbereich Ingenieurwesen/Wirtschaftingenieurwesen, Diplom-Arbeit 1997

\section{Autor}

Prof. Dr.-Ing. Eberhard Wegener

Technische Fachhochschule Wildau Fachbereich Ingenieurwesen/Wirtschaftsingenieurwesen Tel. (0 33 75) 508-169

E-Mail: wegener@vt.tfh-wildau.de 
Tabelle 1: Einflußmöglichkeiten aus dem Projektierungsprozeß für eine montagegerechte Gestaltung verfahrenstechnischer Anlagen

\begin{tabular}{|c|c|c|c|c|c|}
\hline 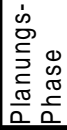 & $\begin{array}{l}\text { Rationalisierungs- } \\
\text { ansätze aus dem } \\
\text { Montageprozeß }\end{array}$ & \multicolumn{3}{|c|}{$\left.\right|_{\text {ntageverrichtungen und -operationen infolge: }} ^{\text {Senkung des Montageaufwandes durch }}$} & $\begin{array}{l}\text { Reduzierung } \\
\text { "montagefrem der" } \\
\text { Leistungen durch }\end{array}$ \\
\hline \multirow{4}{*}{ 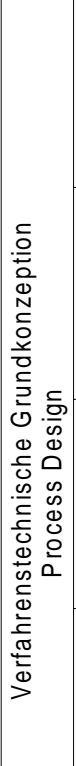 } & $\begin{array}{l}\text { Integralbauweise } \\
\text { (Vereinigung mehrerer } \\
\text { Ausrüstungen oder } \\
\text { Bauelemente zu einer) } \\
\text { Kombination von } \\
\text { Wirkprinzipien }\end{array}$ & $\begin{array}{l}\text { Verringern der Anzahl } \\
\text { von zu montierenden } \\
\text { Bauelementen } \\
\text { insgesamt (absolut) }\end{array}$ & & & \\
\hline & $\begin{array}{l}\text { Zuordnung von } \\
\text { Parameteränderungen } \\
\text { (Ausgleich) durch } \\
\text { Kopplungen zu den } \\
\text { benachbarten } \\
\text { Ausrüstungen }\end{array}$ & $\begin{array}{l}\text { Verringerung der } \\
\text { Anzahl von zu } \\
\text { montierenden } \\
\text { Bauelementen } \\
\text { insgesamt (absolut) }\end{array}$ & & & \\
\hline & $\begin{array}{l}\text { Verringerung der } \\
\text { Ausrüstungsvielfalt } \\
\text { verfahrenstechnisch } \\
\text { abhängiger } \\
\text { Montageeinheiten }\end{array}$ & & $\begin{array}{l}\text { Verbesserung des } \\
\text { Auslastungsgrades } \\
\text { von Arbeitsmitteln } \\
\text { bzw. Deren } \\
\text { Einsatzhäufigkeit }\end{array}$ & & \\
\hline & $\begin{array}{l}\text { Verwendung von } \\
\text { Standardlösungen } \\
\text { für verfahrens- } \\
\text { technisch abhängige } \\
\text { Ausrüstungen }\end{array}$ & & \multicolumn{3}{|c|}{$\begin{array}{l}\text { Erfahrungsrückfluß } \\
\text { Einführung und Anwendung von } \\
\text { Rahmen- bzw. Typentechnologien } \\
\text { in der Montage }\end{array}$} \\
\hline \multirow{4}{*}{ 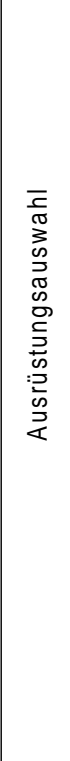 } & $\begin{array}{l}\text { Geometrische } \\
\text { Dimensionierung } \\
\text { nicht-verfahrenstechnisch } \\
\text { abhängiger } \\
\text { Ausurustungen } \\
\text { nach } \\
\text { Optimalitätsktriterien } \\
\text { der Montage }\end{array}$ & & \multicolumn{2}{|c|}{$\begin{array}{l}\text { Transportlage möglichst gleich Einbaulage } \\
\text { Verbesserung der Positionierbarkeit, Lagesicherung } \\
\text { und Ausrichtung } \\
\text { Ausnutzung der Parameter der Hebezeuge und Fördermittel } \\
\text { Vermeidung von Erdstabilisierungsmaßnahmen }\end{array}$} & $\begin{array}{l}\text { Berücksichtigung } \\
\text { des Sonderlastfalles } \\
\text { "Montage" }\end{array}$ \\
\hline & $\begin{array}{l}\text { Verringerung der } \\
\text { Ausrüstungsvielfalt } \\
\text { bei nicht-verfahrens- } \\
\text { technischen } \\
\text { Ausrüstungen }\end{array}$ & $\begin{array}{l}\text { Verbesserung des } \\
\text { Auslastungsgrades } \\
\text { und Einsatz- } \\
\text { häufigkeit der } \\
\text { Arbeitsmittel }\end{array}$ & & & \\
\hline & $\begin{array}{l}\text { Auswahl } \\
\text { genormter } \\
\text { Ausrüstungen }\end{array}$ & & & \multicolumn{2}{|c|}{$\begin{array}{l}\text { Erfahrungsrückfluß } \\
\text { Anwendung von Rahmen- bzw } \\
\text { Typentechnologien in der Montage }\end{array}$} \\
\hline & $\begin{array}{l}\text { Weitgehende } \\
\text { Komplettierung, } \\
\text { Anlieferungsustand } \\
=\mathrm{f}(\text { Einbauzustand })\end{array}$ & $\begin{array}{l}\text { Senkung des } \\
\text { Rüstaufwandes }\end{array}$ & \multicolumn{2}{|c|}{$\begin{array}{l}\text { Vermeidung zusätzlicher Vorbereitungsarbeiten } \\
\text { Vermeidung zusätzlicher Prüf- und Kontrollprozesse } \\
\text { Vermeidung von Spezialgewerken }\end{array}$} & \\
\hline
\end{tabular}


Tabelle 1: Einflußmöglichkeiten aus dem Projektierungsprozeß für eine montagegerechte Gestaltung verfahrenstechnischer Anlagen Fortsetzung

\begin{tabular}{|c|c|c|c|c|}
\hline 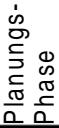 & $\begin{array}{l}\text { Rationalisierungs- } \\
\text { ansätze aus dem } \\
\text { Montageprozeß }\end{array}$ & \multicolumn{2}{|c|}{$\begin{array}{c}\text { Senkung des Montageaufwandes durch } \\
\text { Verringern }\end{array}$} & $\begin{array}{l}\text { Reduzierung } \\
\text { "montagefremder" } \\
\text { Leistungen durch }\end{array}$ \\
\hline \multirow{6}{*}{ 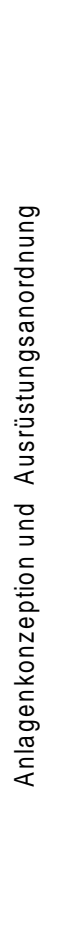 } & $\begin{array}{l}\text { Aufgliederung der } \\
\text { Anlage in Teilanlagen }\end{array}$ & & $\begin{array}{l}\text { Abgrenzung funktionfähiger Teilanlagen, } \\
\text { Untergliederung des Montageprozesses } \\
\text { Verbesserung der Zugänglichkeit } \\
\text { Zweckmäßige Größe/Lage der Baustelleneinrichtung, } \\
\text { Auswahl mobiler Hebezeuge, Fahr- und Montage- } \\
\text { straßen, Vormontageplätze, Auf-, Ab- und Umrüstflächen }\end{array}$ & \\
\hline & $\begin{array}{l}\text { Montagegünstige } \\
\text { Ausrüstungsanordnung }\end{array}$ & & $\begin{array}{l}\text { Gestaltung des Montageraumes, Deckenhöhen, } \\
\text { Montage-, Transport- und Ablageflächen } \\
\text { Verbesserung der Montagebedingungen } \\
\text { und -position }\end{array}$ & \\
\hline & $\begin{array}{l}\text { Nichtüberschreitung } \\
\text { verfahrensbedingter } \\
\text { Höhenunterschiede }\end{array}$ & $\begin{array}{l}\text { Einsparung von } \\
\text { Bauelementen } \\
\text { insgesamt }\end{array}$ & $\begin{array}{l}\text { Beschränkung der Hebezeugparameter } \\
\text { und -stellflächen }\end{array}$ & \\
\hline & $\begin{array}{l}\text { Montagegünstige } \\
\text { Trassenführung }\end{array}$ & & $\begin{array}{l}\text { Sicherung der Zugänglichkeit zum Baufeld } \\
\text { für AM, Montageeinheiten und Transportmittel } \\
\text { Einsatz moderner Montagetechnologien, } \\
\text { insbesondere bei Rohrbrücken }\end{array}$ & $\begin{array}{l}\text { Vermeidung von De- und } \\
\text { Wiederholmontagen, sowie } \\
\text { Umrüstungen von AM }\end{array}$ \\
\hline & $\begin{array}{l}\text { Distanzminimierte } \\
\text { Ausrüstungsanordnung }\end{array}$ & $\begin{array}{l}\text { Einsparung von } \\
\text { Bauelementen insgesamt }\end{array}$ & & \\
\hline & $\begin{array}{l}\text { Zweckmäßige Anordnung } \\
\text { von Schwerst- } \\
\text { ausrüstungen }\end{array}$ & & $\begin{array}{l}\text { Ausnutzung der Tragfähigkeit der Hebezeuge } \\
\text { und des Schwenkbereiches } \\
\text { Vermeidung von Standortwechseln der AM } \\
\text { Gewährleistung genügender Hebezeugfreiheit }\end{array}$ & \\
\hline$\stackrel{\text { O }}{\Xi}$ & $\begin{array}{l}\text { Ausreichende Abstände } \\
\text { zwischwen den } \\
\text { Ausrüstungen }\end{array}$ & & $\begin{array}{l}\text { Gewährleistung des Transportes zum Montageort } \\
\text { und Herstellen der Einbaulage sowie zum } \\
\text { Positionieren }\end{array}$ & $\begin{array}{l}\text { Vermeidung von } \\
\text { Wiederholmontagen }\end{array}$ \\
\hline 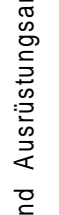 & $\begin{array}{l}\text { Ausreichende Berück- } \\
\text { sichtigung von } \\
\text { Flächen zum } \\
\text { Antransport, } \\
\text { Zwischenlagern und } \\
\text { zum Einbau von } \\
\text { Montageeinheiten }\end{array}$ & & $\begin{array}{l}\text { Berücksichtigung für Montagearbeiten an } \\
\text { Kopplungen und Nachfolgegewerke } \\
\text { Sicherung der Zugänglichkeit }\end{array}$ & $\begin{array}{l}\text { Vermeidung von } \\
\text { Beschädigungen } \\
\text { vorangegangener } \\
\text { Montagearbeiten }\end{array}$ \\
\hline 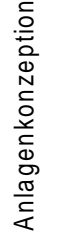 & $\begin{array}{l}\text { Bezug aller Koordinaten } \\
\text { der Einbauorte } \\
\text { auf einen } \\
\text { Festpunkt der } \\
\text { Baustelle }\end{array}$ & & $\begin{array}{l}\text { Senkung der Maßabweichungen und des } \\
\text { Justieraufwandes }\end{array}$ & $\begin{array}{l}\text { Vermeiden mehrfachen } \\
\text { Anpassens }\end{array}$ \\
\hline
\end{tabular}


Tabelle 1: Einflußmöglichkeiten aus dem Projektierungsprozeß für eine montagegerechte Gestaltung verfahrenstechnischer Anlagen Fortsetzung

\begin{tabular}{|c|c|c|c|c|c|}
\hline 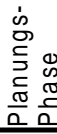 & $\begin{array}{l}\text { Rationalisierungs- } \\
\text { ansätze aus dem } \\
\text { Montageprozeß }\end{array}$ & \multicolumn{3}{|c|}{ Senkung des Montageaufwandes durch } & $\begin{array}{l}\text { Reduzierung } \\
\text { "montagefremder" } \\
\text { Leistungen durch }\end{array}$ \\
\hline \multirow{8}{*}{ 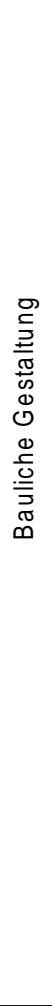 } & $\begin{array}{l}\text { Horizontalbauweise } \\
\text { Freibau } \\
\text { Teilfreibau }\end{array}$ & & \multicolumn{2}{|c|}{$\begin{array}{l}\text { Einfluß auf Einsatz vormontierter und vorgefertigter } \\
\text { Montageeinheiten mit hohem Komplettierungsgrad } \\
\text { Einsatz von Hebezeugen, Zugänglichkeit } \\
\text { Anpassung an benachbarte Ausrüstungen, } \\
\text { Bau- und Stahlbaukonstzruktionen } \\
\text { Komplettierung mit Spezialgewerken }\end{array}$} & \\
\hline & $\begin{array}{l}\text { Kombinierte Bau- } \\
\text { Ausrüstungs-Montage }\end{array}$ & & \multicolumn{2}{|c|}{$\begin{array}{l}\text { Möglichkeit der Erhöhung der Parallelität, } \\
\text { zeitliches und räumliches Nebeneinander } \\
\text { von Montageprozessen }\end{array}$} & \\
\hline & $\begin{array}{l}\text { Stahlskelette } \\
\text { (Gerüstbauweise) als } \\
\text { Tragwerke für die } \\
\text { Ausrüstungen }\end{array}$ & & \multicolumn{2}{|c|}{$\begin{array}{l}\text { Einfache Befestigung von Ausrüstungen, } \\
\text { Rohrleitungen sowie vereinfachtte unkomplizierte } \\
\text { spätere Erweiterung oder Änderung bei } \\
\text { Rekonstruktion } \\
\text { Einfaches Anbringen von Montagehilfsmitteln (MHM) }\end{array}$} & \\
\hline & $\begin{array}{l}\text { Ausreichende Bauwerks- } \\
\text { öfnungen, Montage- } \\
\text { träger, stationäre AM }\end{array}$ & & \multicolumn{2}{|c|}{$\begin{array}{l}\text { Gewährleistung der Anbringung der Montageeinheiten, } \\
\text { Herstellen der Einbaulage, Positionieren }\end{array}$} & \\
\hline & $\begin{array}{l}\text { Einordnung und Gestaltung } \\
\text { der } \mathrm{BE}\end{array}$ & & \multicolumn{2}{|c|}{$\begin{array}{l}\text { Lage- und Flächengliederung ohne Behinderung der } \\
\text { Erweiterung der definierten Anlagen, möglichst geradlinig } \\
\text { geführte Verbindung Montageeinheit -Montageort } \\
\text { Sicherung der notwendigen Vormontage }\end{array}$} & \\
\hline & $\begin{array}{l}\text { Ausreichende Breite und } \\
\text { Profilhöhe der Baustraßen } \\
\text { und Belastbarkeit }\end{array}$ & & \multicolumn{2}{|c|}{$\begin{array}{l}\text { Sicherung des Zuführens maximal vorgefertigter und vormon- } \\
\text { tierter Montageeinheiten und der dazu notwendigen } \\
\text { Transportmittel und Hebezeuge }\end{array}$} & \\
\hline & $\begin{array}{l}\text { Berücksichtigung } \\
\text { des LF "Montage" }\end{array}$ & & \multicolumn{2}{|c|}{$\begin{array}{l}\text { Sicherung der während der der Montage zusätzlich auftre- } \\
\text { tenden Belastungen durch Antransport, zeitliches Abset- } \\
\text { zen, Lastaufnahme über Montageträger, Vorspannkräfte } \\
\text { bzw. Stabilisierung von ME, Prüf- und Kontrollprozesse } \\
\text { Gewährleistung der notwendigen Anschlagpunkte für die } \\
\text { Krafteinleitung in den Baukörper für Hebezeuge, Träger } \\
\text { Stützen, Ösen, Verankerungen }\end{array}$} & \\
\hline & $\begin{array}{l}\text { Zweckmäßige Tolerierung } \\
\text { und Achsenmarkierung }\end{array}$ & & \multicolumn{2}{|c|}{$\begin{array}{l}\text { Reduzierung von Justierarbeiten } \\
\text { Sicherung des Positionierens und Ausrichtens }\end{array}$} & \\
\hline \multirow{9}{*}{ 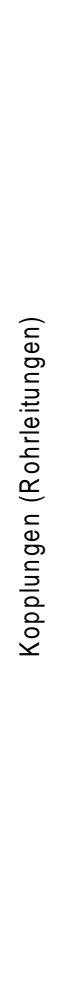 } & $\begin{array}{l}\text { Anwendung der Differen- } \\
\text { tialbauweise (Elementari- } \\
\text { sierung durch konstruktove } \\
\text { Aufteilung der Rohrleitung }\end{array}$ & & \multicolumn{2}{|c|}{$\begin{array}{l}\text { Verringerung der am Einbauort zu montierenden } \\
\text { Bauelementezahl durch zentrale oder dezentrale } \\
\text { Vorfertigung }\end{array}$} & $\begin{array}{l}\text { Berücksichtigung des } \\
\text { Lastfalles Montageund } \\
\text { des Montagefortschritts }\end{array}$ \\
\hline & $\begin{array}{l}\text { Überprüfen von Verbindun- } \\
\text { gen auf Niotwendigkeit, } \\
\text { wenn aus funktionell oder } \\
\text { aus GABS-Gründen nicht } \\
\text { bedingt }\end{array}$ & $\begin{array}{l}\text { Verringerung der } \\
\text { Bauelementezahl } \\
\text { insgesamt }\end{array}$ & & & \\
\hline & $\begin{array}{l}\text { Überprüfen von Verbindun- } \\
\text { gen auf Zweckmäßigkeit } \\
\text { in der Ausführungn }\end{array}$ & & \multicolumn{2}{|c|}{$\begin{array}{l}\text { Zugänglichkeit für Arbeitskräfte und Arbeitsmittel } \\
\text { Einsatz rationeller Fügeverfahren }\end{array}$} & \\
\hline & $\begin{array}{l}\text { Zweckmäßige Anordnung } \\
\text { der Rohrleitungen }\end{array}$ & & \multicolumn{2}{|c|}{$\begin{array}{l}\text { Zugänglichkeit für Arbeitskräfte und Arbeitsmittel } \\
\text { Einsatz rationeller Montagetechnologien und Fügeverfahren } \\
\text { Erleichterung der Prüf- und Kontrollprozesse }\end{array}$} & \\
\hline & Systeme gleichen Druckes & & & $\begin{array}{l}\text { Erleichterung und Aufwands- } \\
\text { senkung für Kontrollprozesse }\end{array}$ & \\
\hline & $\begin{array}{l}\text { Zweckm. Anordnung und } \\
\text { Gestaltung der Unter- } \\
\text { stützungselemente }\end{array}$ & & \multicolumn{2}{|c|}{$\begin{array}{l}\text { Gewährleistung der Zugänglichkeit } \\
\text { Sicherung des Positionierens }\end{array}$} & \\
\hline & $\begin{array}{l}\text { Zweckmäßige Anordnung } \\
\text { von Armaturen und } \\
\text { Montagehilfseinrichtungen }\end{array}$ & & \multicolumn{2}{|c|}{$\begin{array}{l}\text { Gewahrleistung der Zugänglichkeit } \\
\qquad \begin{array}{l}\text { Erleichterung der Montage/ } \\
\text { Demontage }\end{array}\end{array}$} & \\
\hline & $\begin{array}{l}\text { Ausreichende und zweck- } \\
\text { mäßige Anordnung von } \\
\text { Bühnen, Laufstegen u.a. } \\
\text { Bedienungs- und Montage- } \\
\text { flächen }\end{array}$ & & \multicolumn{2}{|c|}{$\begin{array}{l}\text { Gewährleistung der Zugänglichkeit } \\
\text { Sicherung der Montage-und Abstellflächen } \\
\text { Gewährleistung des Einsatzes rationeller Füge- } \\
\text { verfahren und Prüftechnologien }\end{array}$} & \\
\hline & $\begin{array}{l}\text { Zweckmäßige Kompen- } \\
\text { sation von Maßab- } \\
\text { weichungen }\end{array}$ & & $\begin{array}{l}\text { Reduzierung von } \mathrm{Paßarbeiten} \\
\text { Senkung operativer Lösungen }\end{array}$ & & $\begin{array}{l}\text { Vermeidung bzw. } \\
\text { Verminderung von } \\
\text { Wiederholmontagen } \\
\text { und Neuanferti- } \\
\text { gungen }\end{array}$ \\
\hline
\end{tabular}


Tabelle 1: Einflußmöglichkeiten aus dem Projektierungsprozeß für eine montagegerechte Gestaltung verfahrenstechnischer Anlagen Fortsetzung

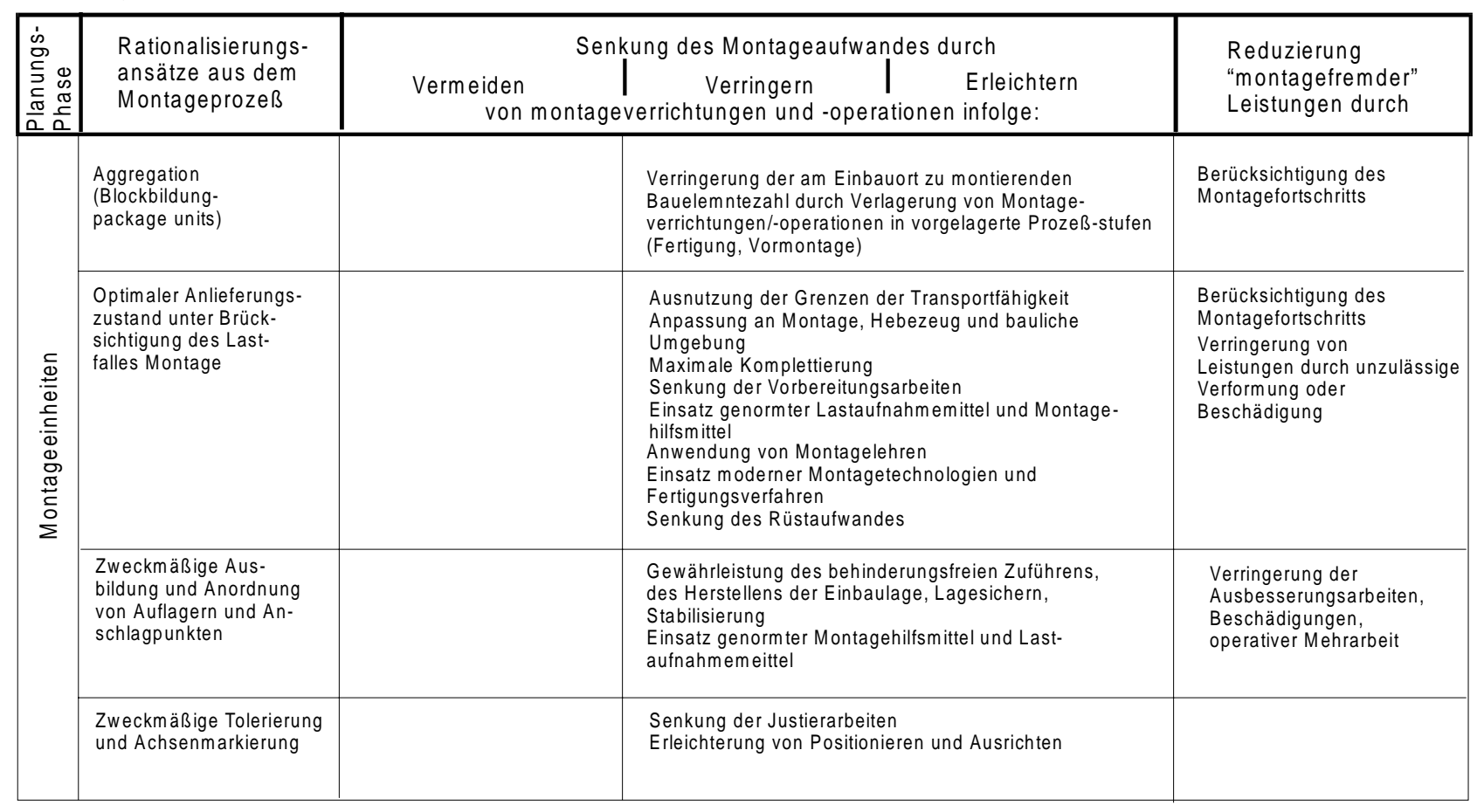

